\title{
Student wellness at home and school: A holistic perspective
}

\section{Sandra Moynan-Longworth}

$\Gamma$ he holistic approach to wellness is to teach chil dren and adults how to "Thrive" rather than just, "Survive". Teachers may be interested in using some of what follows to create lessons that inform students and parents about holistic approaches to wellness.

\section{Lifestyle Wellness}

\section{Exercise}

Parents and children must learn and understand that exercise is critical to good health. It increases bone density and muscle mass. It helps lower the level of glucose circulating in the blood and increases insulin receptors on the cells. Exercise increases blood flow to the brain and to every organ and cell in the body. Research demonstrates that appropriate exercise results in improved school performance, enhanced attitude, and contributes to positive self-esteem, wellness and resilience. Aside from scheduled PE classes, teachers can implement a daily exercise program both in and out of the classroom.

\section{Rest}

Children's bones, glands, organs and muscles go through tremendous development stress and require down time for repair and rejuvenation. Too little rest causes the developmental processes to break down and can cause serious health and emotional risks. A lack of rest can lead to poor health, lowered academic performance,

irritability and disruptive behaviour. If teachers suspect that students are not well rested they should discuss this with parents.

It is important that we teach children that caring for self and the environment begins at home and at school.

\section{Diet}

A variety of poorly managed environmental factors and the quality of foods consumed contribute to overall ill health. Research clearly links consumption of certain food types to learning and behavioural difficulties and to the onset of various health issues. Chemicals and artificial ingredients used in foods, natural or processed, restrict our intake of natural vitamins and minerals. Individuals who lead stressful and demanding lives require supplements to ensure a healthy lifestyle and overall wellness.

Breakfast is the most important meal of the day. After going without food for eight or more hours, a child needs a nutritional boost to get started in the morning (they are "breaking" the "fast", literally). Many studies show that children whose breakfast is nutritionally inadequate demonstrate lower academic and behavioural performance across a broad spectrum of tasks.

Students will benefit from knowing what a healthy diet is and how to maintain it. Teachers can investigate ways to include lessons about diet across a broad spectrum of the school curriculum. For instance children and adolescents should know that good dietary habits should include eating fowl with skin removed, ocean fish rather than farm fed varieties, low-fat dairy products, all the veggies they want, lots of fruit, legumes, such as beans, lentils, peas etc., whole grain products - pasta, cereal, raw nuts and seeds and cold-pressed vegetable oils. Children should learn to choose foods made with whole, natural ingredients and know what safe sweeteners are such as stevia (found at your local health food store), honey, molasses, pure maple syrup and fructose.

Other points worth noting are that sea salt is better than table salt, roasted nuts and seeds should be avoided and of course chips and other snack foods high in fat are unhealthy to say the least. Health food culture often refers to fish products as brain food. It might be interesting to share with students that by eating fish products that contain fatty acids such as Omega 3-6-9 it may increase their brain power and possibly help students become better learners. Some argue that Omegas also promote hormone balance. This is good for teens and may even be the basis for reducing stressful moments at home and in the classroom.

Vitamin supplements are an important nutritional benefit. Sugar coated or food coloured vitamins are not recommended. Preferably vitamins and minerals that are manufactured with natural, unaltered food concentrate methods and contain active en- 
zymes are best. Vitamins improve bone density, help immune systems to fight of disease and colds and contribute to overall well being and may increase learning power.

Critical to diet is appropriate water intake. Active children and teens lose copious amounts of water through normal activity and much more during high activity periods. Water is essential for good circulation, assimilation, of nutrients, detoxification, temperature control and effective digestion and elimination. Students should be encouraged to drink plenty of water throughout the day. In fact, teachers might consider encouraging regular water or juice breaks. Many patients wonder if purified water is better than tap water. The debate is interesting and space does not warrant an appropriate discussion. It is important however to understand that chlorine and other additives used to kill bacteria is toxic and can lead to a variety of health hazards. Effective water filtration through simple add on filters or osmosis systems can help to ensure a clean and safe water supply. It is important to check if bottled water purchased at a convenience or grocery store in actually filtered water. Students also need to know that coffee and tea, alcohol stimulated products and high energy juices and stimulant drinks are not preferred forms of liquid intake and can be harmful, certainly detrimental to health.

\section{Toxin Exposure}

Children, especially the very young, are more vulnerable to toxins. They breathe in two or three times as much air (and therefore pollutants) relative to body size. Exposure to toxins comes from contact with cleaners used in homes and schools, even hospitals. Many of which are known to be carcinogenic. More than ten thousand children in Canada have some form of Cancer. The body accumulates toxins and although it has its own detoxification system, in some instances, it cannot keep up with the bombardment of exposure to toxins and the result is a weak immune system. Teachers can inform students about how to reduce toxin load and exposure. Statistics show that $70 \%$ of soil and groundwater contamination in North America comes from "household cleaners, laundry products, and alike. According to Dr. Doris Rapp, a medical doctor who specializes in Environmental Medicine, "The accumulation of chemicals in the human body causes a long list of major health problems: learning disabilities, chronic asthma, headaches, skin problems, mood swings, anxiety and inappropriate behaviour. It affects the brain so dramatically that it causes irritability, the inability to think clearly, memory loss, and poor coordination."

Students will benefit from knowing what a healthy diet is and how to maintain it. Teachers can investigate ways to include lessons about diet across a broad spectrum of the school curriculum.

\section{Allergies and Asthma}

One cannot talk about healthy diet and environmental conditions that contribute to ill health without referring to allergies and asthma. These are often called the hidden disabilities. Both can be attributed to serious other health concerns and have been shown to contribute significantly to a variety of school learning difficulties and behavioural outcomes.
Food allergies can be described in terms of two distinct categories. They are genetic and acquired. In each case if a child is suspected of an allergy it is important to recommend to parents that the child be tested. The Elisa Rast Blood Allergy test is a simple non invasive method that effectively discovers more than one hundred food and fifty inhalant reactions.

\section{Air Quality}

Schools are notorious for poor air quality. Many children and teachers react, sometimes severely, to mould, dust mites, pet dander, bacteria, pollen, dust, and chemical fumes. Research supports what many have known, that is, often air quality indoors can often be significantly worse than outdoor air. It is known that just by breathing polluted indoor air an individual's risk of lung cancer can increase significantly. Chronic sinusitis is often related to poor indoor air quality as are headaches, sneezing, itchy eyes, lack of alertness and general drowsiness. Opening the windows in a classroom is a short term solution however it is imperative that air quality be tested on a regular basis and that filters be installed. Quality air is an important step in removing irritants that stimulate allergic reactions.

Food allergies can be lethal and teachers should be well informed about individual cases. In any event a list of known allergy foods should be posted in all classrooms and cafeteria areas. It is important that teachers, staff and students are informed about what to do in the event of severe reaction. While proactive intervention is the key, first line crisis intervention can often save a life. Schools should strive to continually educate staffs about policies and procedures that 


\section{3:1 Winter 2005}

affect students and teachers with allergies.

Asthma is also a serious debilitating health concern. Death rates due to asthma and bronchitis have risen considerably in the last ten years. It is estimated that in Ontario alone, 12,000 children are hospitalized with asthma every year. Asthma is one of the single most frequent reasons why children ate hospitalized. More and more children are prescribed inhalers containing a muscle relaxant medication that can provide immediate relief during a severe attack. The problem is that while helpful it does not treat the problem it basically masks or suppresses the condition. Prolonged use can lead to reduced immune and endocrine functions.

Like an allergic attack an asthma attack is often triggered by a reaction to an inhalant or a food. Likewise the same offenders known to allergy sufferers can induce an asthma attack or spasm. Well known pain medication such as Aspirin, Ibuprofen, Advil, Nuprin, Naproxen, and others have been directly linked to many severe asthmatic reactions. Asprin being the most likely culprit. Mineral and vitamin deficiencies particularly Vitamin C, E and B complex also contribute to the condition. Children and teens diagnosed with weak adrenal glands are often treated for asthma symptoms with corticosteroids. Even cold water can in some instances trigger an attack. Repeated attacks are energy draining and contribute to a lack of motivation and willingness to attend to school related tasks. Children and teens need to be encouraged to understand what it means to have an allergy or asthma and how it may affect their overall wellness and performance in school. Teachers should be empathetic towards students who exhibit symptoms and provide opportunities for students to complete unfinished assignments etc. without penalty.

Food allergies can be lethal and teachers should be well informed about individual cases. In any event a list of known allergy foods should be posted in all classrooms and cafeteria areas.

\section{Helpful Strategies}

While teachers may not be able to control diet and environmental factors they can educate students and parents about the importance of understanding the affects of allergies and asthma on learning and other school related behaviours. Teachers can display posters, talk with sufferers, modify lessons and assignment workloads, and case manage students through team meetings and information sharing. It is essential that students be made aware that a proper diet, appropriate exercise and rest are imperative ingredients to well being. The Canada Food Guide is a good starting point and there is much information available to teachers from a variety of healthy consumer advocates. Interested teachers can instruct students about alternative natural food options and they can motivate and reward students who explore and share these options. Any health food outlet but particularly nutritionists and holistic practitioners will gladly assist teachers with resources and information about food supplements, vitamins and minerals, food combinations and herbal remedies that help reduce conditions associated with allergy and asthma.

\section{Touch Therapy}

As a holistic Practitioner and Natural Therapist I use many bodywork treatment therapies. The most helpful with children and teens are Acupuncture, Aromatherapy and Lymphatic massage. When I treat children with a massage therapy technique it acts as a great "door opener." Through compassionate touch, comfort and trust the action of "touch" invites and promotes therapeutic communication and exchange. Aromatherapy is the use of essential oils, which are pure, unadulterated, plant extracts, derived from flowers, herbs, fruits and trees, to reduce stress, promote relaxation and aid the body in healing itself. Non invasive, lymphatic drainage massage techniques stimulate the sense of smell and absorption of the oils to relax the client and encourage the lymphatic system to work more effectively by eleminating toxins and boosting the immune system.

\section{Acupuncture}

Acupuncture is one of the oldest and most powerful methods of treating acute and chronic pain by activating the body's natural powers of self-healing. Children, not to mention some grown ups, are sometimes frightened by needles. Once I have had a chance to get to know them, explain the process and show them the "hair thin" needles, they usually relax. Although Acupuncture is useful for a variety of health problems, including addiction and mental disorder, its' most common use is to relieve migraine headaches, back pain and inflammation. It involves the insertion of 5 to 20 very fine needles at different points on the body to stimulate the flow of energy, also called "chee". I often recommend 
herb supplements and suggest specific lifestyle changes and exercises.

\section{Reiki}

I find that children are so open to this wonderful treatment approach. Reiki, pronounced "RayKey" dates its origin to ancient Tibet. REI means "universal" and it refers to the spiritual dimension and the soul. KI means the "vital life force" energy, which flows through all that lives. Reiki energy comes from the Universal Life Force and is an extremely powerful healing energy. This non-invasive therapy treatment is practiced by Reiki masters trained in the art of energy force healing. Simply by placing one's hands a few inches above the body, the energy flows through the practitioner's hands and into the child receiving the healing. Reiki light energy therapy is a wonderful alternative to mainstream muscle manipulation therapies and affords patients a pleasant, healing energy enhancement intervention treatment for pain due to strain, injury, tension and stress.

\section{In Closing}

Psychological well being is extremely important to overall wellness. And, similar to physical well being, an individual's overall sense of balance and harmony requires constant attention. Parents and teachers should be mindful that a child's body requires a synergistic and harmonious interplay of both aspects of development in order to develop resilience and well being.

This article illustrates the relationship between learning and behaviour in school and students who experience severe and or chronic allergy or asthma symptoms.
Teachers too, are susceptible to the same irritants and can also be affected by poor diet, breathing polluted air and drinking unsafe water. Children need teachers to constantly reinforce the fact that a good diet is essential to good health and that there are alternatives they need to know about, understand and explore. Despite what might be said that a big Mac and a coke are reasonably nutritious alternatives the fact that child and teen obesity can be linked to fast food alternatives clearly suggests that teachers must be leaders.

Healthy living requires work and effort. Teachers are well situated to be role models. In fact it is an expectation. From my perspective teachers are needed today, more than ever. Not only to extrapolate the increasingly complex curriculum of our technological age but to enhance student learning through an examination of what it means to live a healthy and safe life and to promote an environmentally friendly living space. Whether we like it or not the choices we make individually and collectively make a difference in not only our own lives but the lives of others. Teachers and parents must continue to work together to provide healthy living environments for children. As a holistic professional it is my chosen responsibility to promote and provide healing strategies and techniques that help make wellness an obtainable goal for children and adults.

\section{Suggested readings and references}

Cave, Stephanie, M.D. What your doctor may NOT tell you about children's vaccinations. ISBN 044661503x. Time Warner Books.
Diodati, Catherine J.M., M.A. Immunization History, ethics, law and health. ISBN 0968508006 Integral Aspects Inc., diodati@mnsi.net

McDougall, M.D. and McDougall, Mary A., The McDougall Plan. Available through the McDougall Wellness Center. drmcdougall@drmcdougall.com.

Rapp, Dr. Doris, FAAA, FAAP Is this your child. Available through living well naturally. www.thebook.com/ live well.

Robbins, John. The Food Revolution. Available through begsource.com and John Robbins home page.

Sandy Moynan-Longworth is a member of the Canadian Examining

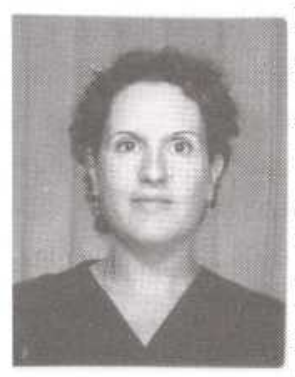
Board of Regis$t$ e $r$ e d Aromatherapists and the Association of Natural Therapists and Naturopaths. She is a practicing Doctor of Acupuncture, CCCA, certified Cosmetic Acupuncturist (non surgical face lifting) registered Aromatherapist, certified Wellness consultant and Symptomologist, a Lymph drainage Massage therapist and a certified Early Childhood Education Teacher. In addition Sandra Moyan Longworth is a Reiki Master. Sandy provides holistic treatment and therapy to adults and children and is dedicated to educating and treating individuals and families about healthy living and the power of natural healing by addressing the source not just the symptoms. For consultation and or information Contact Sandy at Aldershot, Ontario, Canada, 905-681-9491 or smlongworth@sympatico.ca. 